\title{
HAUSDORFF DISTANCE BETWEEN THE OFFSET CURVE OF QUADRATIC BEZIER CURVE AND ITS QUADRATIC APPROXIMATION
}

\author{
Young JoON AhN
}

\begin{abstract}
In this paper, we present the exact Hausdorff distance between the offset curve of quadratic Bézier curve and its quadratic $G C^{1}$ approximation. To illustrate the formula for the Hausdorff distance, we give an example of the quadratic $G C^{1}$ approximation of the offset curve of a quadratic Bézier curve.
\end{abstract}

\section{Preliminaries}

Quadratic Bézier curves and their offset curves are widely used in CAD/CAM or Computer Graphics. But offset curve of quadratic Bézier curve cannot be expressed in Bézier form. In the recent twenty years, many papers about the quadratic Bézier approximation $[2,7,13]$ or the offset curve $[6,8,10,11,15]$ have been published. Especially as the error measurement between the target curve and approximation curve, the Hausdorff distance is used in CAD/CAM or Approximation Theory. The Hausdorff distance $d_{H}(\mathbf{p}, \mathbf{q})$ between two curves $\mathbf{p}(s), s \in[a, b]$ and $\mathbf{q}(t), t \in[c, d]$, is given by

$$
d_{H}(\mathbf{p}, \mathbf{q})=\max \left\{\max _{s \in[a, b]} \min _{t \in[c, d]}|\mathbf{p}(s)-\mathbf{q}(t)|, \max _{t \in[c, d]} \min _{s \in[a, b]}|\mathbf{p}(s)-\mathbf{q}(t)|\right\} .
$$

(For more knowledge about the Hausdorff distance, refer to $[1,14,12]$ ) By the way, it is not easy to find the Hausdorff distance between planar curve and quadratic Bézier curve. The Hausdorff distance is obtained from the maximum distance between $\mathbf{p}\left(s_{0}\right)$ and $\mathbf{q}\left(t_{0}\right)$ satisfying

$$
\mathbf{p}^{\prime}\left(s_{0}\right) \| \mathbf{q}^{\prime}\left(t_{0}\right) \quad \text { and } \quad \mathbf{p}^{\prime}\left(s_{0}\right) \perp \overrightarrow{\mathbf{p}\left(s_{0}\right) \mathbf{q}\left(t_{0}\right)}
$$

when they have the same end points, as shown in Figure 1. Thus to get the Hausdorff distance it requires to solve nonlinear system of two variables such as in Equation (1.1).

Received July 11, 2007.

2000 Mathematics Subject Classification. 41A05, 41A15, 65D05, 65D07, 65D17.

Key words and phrases. quadratic Bézier curve, Hausdorff distance, offset curve, control polygon, approximation, error, geometric continuity. 


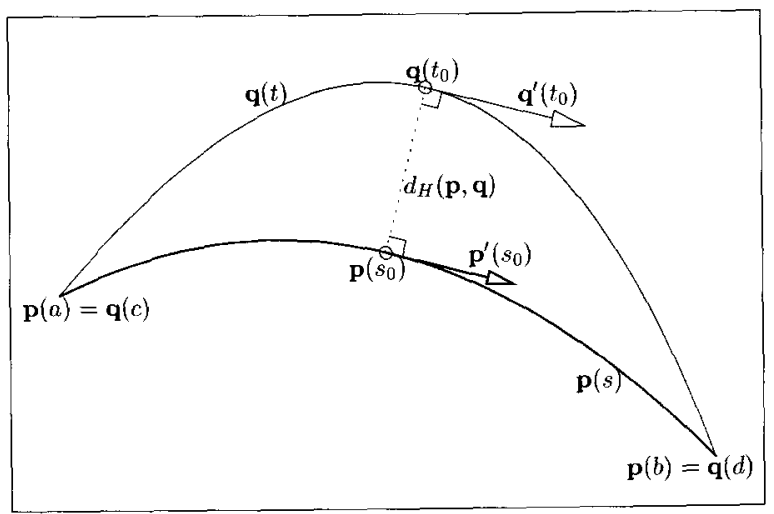

Figure 1. Hausdorff distance between two curves $\mathbf{p}(s), s \in$ $[a, b]$, (thick lines) and $\mathbf{q}(t), t \in[c, d]$ (thin lines).

In this paper we find the formula for the Hausdorff distance between the offset of quadratic Bézier curve and the quadratic $G C^{1}$ Bézier approximation. Using the formula for the Hausdorff distance, we can easily obtain the error of the quadratic $G C^{1}$ Bézier approximation of the offset of quadratic Bézier curve.

\section{Hausdorff distance between offset of quadratic Bézier curve and its quadratic approximation}

In this section we present the error of the quadratic $G C^{1}$ (geometric continuity of order one) end-points interpolation of offset curve of quadratic Bézier curve.

Let $\mathbf{b}(t)$ be the quadratic Bézier curve with the control points $\mathbf{b}_{0}, \mathbf{b}_{1}$ and $\mathbf{b}_{2}$, i.e.,

$$
\mathbf{b}(t)=\sum_{i=0}^{2} B_{i}^{n}(t) \mathbf{b}_{i} \quad t \in[0,1]
$$

(refer to [9]) where $B_{i}^{n}(t)$ is the Bernstein polynomial of degree $n$, i.e.,

$$
B_{i}^{n}(t)=\left(\begin{array}{c}
n \\
i
\end{array}\right) t^{i}(1-t)^{n-i}
$$

Let $T(t), N(t)$ and $k(t)$ be the unit tangent, unit normal vector and curvature of $\mathbf{b}(t)$, in order, i.e.,

$$
T(t)=\mathbf{b}(t) /|\mathbf{b}(t)|, \quad N(t)=T^{\prime}(t) /\left|T^{\prime}(t)\right|, \quad k(t)=\left|\mathbf{b}^{\prime}(t) \times \mathbf{b}^{\prime \prime}(t)\right| /\left|\mathbf{b}^{\prime}(t)\right|^{3} .
$$

Let $N_{i}=N(i)$ for $i=0,1$. We denote the offset curve of $\mathbf{b}(t)$ for the offset distance $d \in \mathbb{R}$ by $\mathbf{b}^{d}(t)$, i.e.,

$$
\mathbf{b}^{d}(t)=\mathbf{b}(t)+d N(t) \quad t \in[0,1]
$$




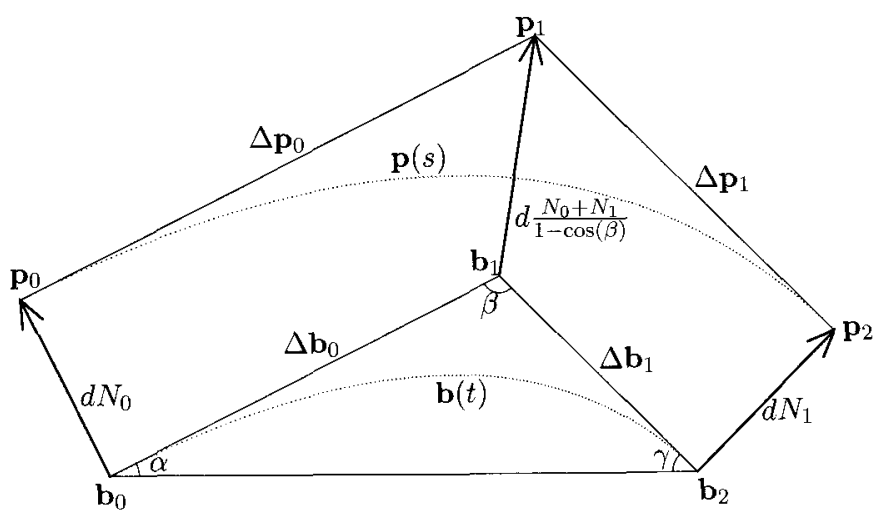

FIGURE 2. Quadratic $G C^{1}$ interpolation $\mathbf{p}(s)$ of offset of quadratic Bézier curve $\mathbf{b}(t)$ with offset distance $d$. The quadratic Bézier curves (dotted lines) $\mathbf{b}(t)$ and $\mathbf{p}(s)$ have the control polygon $\mathbf{b}_{0} \mathbf{b}_{1} \mathbf{b}_{2}$ and $\mathbf{p}_{0} \mathbf{p}_{1} \mathbf{p}_{2}$, respectively.

We assume that the control points are not collinear. Note [10] that if

$$
\min (1 / k(t))>d,
$$

then offset has neither cusp nor loop and the same tangent direction with $\mathbf{b}(t)$ at end points. The necessary and sufficient condition that the quadratic Bézier curve has the local extreme of curvature is well-known [4].

In the following proposition the control polygon of the quadratic $G C^{1}$ endpoints interpolation $\mathbf{p}(s)=\sum_{i=0}^{2} B_{i}^{2}(s) \mathbf{p}_{i}$ of the offset curve $\mathbf{b}^{d}(t)$ is obtained explicitly.

Proposition 2.1. For $d<\min (1 / k(t))$, the quadratic Bézier curve $p(t)$ having the control points

$$
\begin{aligned}
& \mathbf{p}_{0}=\mathbf{b}_{0}+d N_{0} \\
& \mathbf{p}_{1}=\mathbf{b}_{1}+d \frac{N_{0}+N_{1}}{1+N_{0} \circ N_{1}} \\
& \mathbf{p}_{2}=\mathbf{b}_{2}+d N_{1}
\end{aligned}
$$

is $G C^{1}$ end-points interpolation of the offset curve $\mathbf{b}^{d}(t)$.

Proof. It suffices to show that two quadratic Bézier curve $\mathbf{p}(t)$ and $\mathbf{b}(t)$ have the same tangent direction at both end points. For simplification, let $\Delta \mathbf{b}_{i}=$ $\mathbf{b}_{i+1}-\mathbf{b}_{i}$, as shown in Figure 2 .

$$
\Delta \mathbf{p}_{0}=\mathbf{p}_{1}-\mathbf{p}_{0}=\Delta \mathbf{b}_{0}+\frac{d}{1+N_{0} \circ N_{1}}\left(N_{1}-N_{0}\left(N_{0} \circ N_{1}\right)\right) \text {. }
$$


Since $N_{1}-N_{0}\left(N_{0} \circ N_{1}\right)$ is the same direction with $T(0), \Delta \mathbf{p}_{0}$ and $\Delta \mathbf{b}_{0}$ have the same direction. Thus $\mathbf{p}(t)$ and $\mathbf{b}(t)$ have the same tangent direction at $t=0$. By same reason, $\mathbf{p}(t)$ and $\mathbf{b}(t)$ also have the same tangent direction at $t=1$.

The angles in the triangle $\triangle \mathbf{b}_{0} \mathbf{b}_{1} \mathbf{b}_{2}$ are denoted by $\alpha, \beta$ and $\gamma$, in order, as shown in Figure 2. If $\beta=\pi / 2$, then $\mathbf{p}_{1}=\mathbf{b}_{1}+d\left(N_{0}+N_{1}\right)$, if $\beta \rightarrow 0$ then $\mathbf{p}_{1} \rightarrow \infty$, and if $\beta$ tends to $\infty$ then $\mathbf{p}_{1}$ tends to $\mathbf{b}_{1}+d N_{0}$.

Now, we will find all the solutions $(t, s)$ of the equations

$$
\begin{gathered}
\mathbf{b}^{\prime}(t) \| \mathbf{p}^{\prime}(s) \\
\mathbf{b}^{\prime}(t) \perp \overrightarrow{\mathbf{p}(s) \mathbf{b}(t)} .
\end{gathered}
$$

Let $\mathbf{b}(t)=(x(t), y(t)), \mathbf{p}(s)=(u(s), v(s))$. Equation (2.1) yields

$$
u^{\prime}(s) y^{\prime}(t)=v^{\prime}(s) x^{\prime}(t)
$$

Using $\Delta u_{0} \Delta y_{0}=\Delta v_{0} \Delta x_{0}$ and $\Delta u_{1} \Delta y_{1}=\Delta v_{1} \Delta x_{1}$, we have the simple equation

$$
s=\frac{\left(\Delta u_{0} \Delta y_{1}-\Delta v_{0} \Delta x_{1}\right) t}{\left(-\Delta u_{1} \Delta y_{0}+\Delta v_{1} \Delta x_{0}\right)(1-t)+\left(\Delta u_{0} \Delta y_{1}-\Delta v_{0} \Delta x_{1}\right) t}
$$

Let $E_{1}=\Delta u_{0} \Delta y_{1}-\Delta v_{0} \Delta x_{1}$ and $E_{2}=\Delta u_{0} \Delta y_{1}-\Delta v_{0} \Delta x_{1}$. Then $s=s(t)=$ $\frac{E_{1} t}{E_{2}(1-t)+E_{1} t}, s(0)=0$ and $s(1)=1$. Equation (2.2) yields

$$
\begin{aligned}
& (u(s)-x(t), v(s)-y(t)) \circ\left(x^{\prime}(t), y^{\prime}(t)\right)=0 \quad \text { or } \\
& x^{\prime}(t)(u(s)-x(t))+y^{\prime}(t)(v(s)-y(t))=0 .
\end{aligned}
$$

By $1-s(t)=\frac{E_{2}(1-t)}{E_{2}(1-t)+E_{1} t}$, we have

$$
u(s(t))=\frac{E_{2}^{2}(1-t)^{2} u_{0}+2 E_{1} E_{2} t(1-t) u_{1}+E_{1}^{2} t^{2} u_{2}}{\left(E_{2}(1-t)+E_{1} t\right)^{2}}
$$

and

$$
\begin{aligned}
u(s(t))-x(t)= & \frac{1}{\left(E_{2}(1-t)+E_{1} t\right)^{2}}\left(E_{2}^{2}(1-t)^{2} u_{0}\right. \\
& \left.+2 E_{1} E_{2} t(1-t) u_{1}+E_{1}^{2} t^{2} u_{2}-\left(E_{2}(1-t)+E_{1} t\right)^{2} x(t)\right) .
\end{aligned}
$$

The dominator in the last equation is a quartic polynomial, so that it can be expressed in quartic Bézier form as follows.

$$
\begin{aligned}
u(s(t))-x(t)= & \frac{1}{\left(E_{2}(1-t)+E_{1} t\right)^{2}}\left[E_{2}^{2}\left(u_{0}-x_{0}\right) B_{0}^{4}(t)+F_{1} B_{1}^{4}(t)\right. \\
& \left.+F_{2} B_{2}^{4}(t)+F_{3} B_{3}^{4}(t)+E_{1}^{2}\left(u_{2}-x_{2}\right) B_{4}^{4}(t)\right]
\end{aligned}
$$


where

$$
\begin{aligned}
F_{1} & =\frac{1}{2} E_{2}\left(E_{2}\left(u_{0}-x_{1}\right)+E_{1}\left(u_{1}-x_{0}\right)\right) \\
F_{2} & =\frac{1}{6}\left(E_{2}^{2}\left(u_{0}-x_{2}\right)+4 E_{1} E_{2}\left(u_{1}-x_{1}\right)+E_{1}^{2}\left(u_{2}-x_{0}\right)\right) \\
F_{3} & =\frac{1}{2} E_{1}\left(E_{1}\left(u_{2}-x_{1}\right)+E_{2}\left(u_{1}-x_{2}\right)\right)
\end{aligned}
$$

Since $x^{\prime}(t)=2(1-t) \Delta x_{0}+2 t \Delta x_{1}$ and $y^{\prime}(t)=2(1-t) \Delta y_{0}+2 t \Delta y_{1}$, we obtain the following equation in quintic Bézier form

$$
\begin{aligned}
& x^{\prime}(t)(u(s(t))-x(t))\left(E_{2}(1-t)+E_{1} t\right)^{2} \\
= & \left.2 \Delta x_{0} E_{2}^{2}\left(u_{0}-x_{0}\right) B_{0}^{5}(t)+\frac{2}{5}\left[4 \Delta x_{0} F_{1}+\Delta x_{1} E_{2}^{2}\left(u_{0}-x_{0}\right)\right)\right] B_{1}^{5}(t) \\
& +\frac{1}{5}\left[6 \Delta x_{0} F_{2}+4 \Delta x_{1} F_{1}\right] B_{2}^{5}(t)+\frac{1}{5}\left[4 \Delta x_{0} F_{3}+6 \Delta x_{1} F_{2}\right] B_{3}^{5}(t) \\
& +\frac{2}{5}\left[\Delta x_{0} E_{1}^{2}\left(u_{2}-x_{2}\right)+4 \Delta x_{1} F_{3}\right] B_{4}^{5}(t)+2 \Delta x_{1} E_{1}^{2}\left(u_{2}-x_{2}\right) B_{5}^{5}(t) .
\end{aligned}
$$

By same way, we also get the following equation

$$
\begin{aligned}
& y^{\prime}(t)(v(s(t))-y(t))\left(E_{2}(1-t)+E_{1} t\right)^{2} \\
= & \left.2 \Delta y_{0} E_{2}^{2}\left(v_{0}-y_{0}\right) B_{0}^{5}(t)+\frac{2}{5}\left[4 \Delta y_{0} G_{1}+\Delta y_{1} E_{2}^{2}\left(v_{0}-y_{0}\right)\right)\right] B_{1}^{5}(t) \\
& +\frac{1}{5}\left[6 \Delta y_{0} G_{2}+4 \Delta y_{1} G_{1}\right] B_{2}^{5}(t)+\frac{1}{5}\left[4 \Delta y_{0} G_{3}+6 \Delta y_{1} G_{2}\right] B_{3}^{5}(t) \\
& +\frac{2}{5}\left[\Delta y_{0} E_{1}^{2}\left(v_{2}-y_{2}\right)+4 \Delta y_{1} G_{3}\right] B_{4}^{5}(t)+2 \Delta y_{1} E_{1}^{2}\left(v_{2}-y_{2}\right) B_{5}^{5}(t),
\end{aligned}
$$

where

$$
\begin{aligned}
G_{1} & =\frac{1}{2} E_{2}\left(E_{2}\left(v_{0}-y_{1}\right)+E_{1}\left(v_{1}-y_{0}\right)\right) \\
G_{2} & =\frac{1}{6}\left(E_{2}^{2}\left(v_{0}-y_{2}\right)+4 E_{1} E_{2}\left(v_{1}-y_{1}\right)+E_{1}^{2}\left(v_{2}-y_{0}\right)\right) \\
G_{3} & =\frac{1}{2} E_{1}\left(E_{1}\left(v_{2}-y_{1}\right)+E_{2}\left(v_{1}-y_{2}\right)\right) .
\end{aligned}
$$

Since $\Delta x_{0}\left(u_{0}-x_{0}\right)+\Delta y_{0}\left(v_{0}-y_{0}\right)=\Delta x_{1}\left(u_{2}-x_{2}\right)+\Delta y_{1}\left(v_{2}-y_{2}\right)=0$, the coefficients combined with $B_{0}^{5}(t)$ and $B_{5}^{5}(t)$ of $x^{\prime}(t)(u(s(t))-x(t))+y^{\prime}(t)(v(s(t))-$ $y(t))$ are zero. Thus it has the factor $t(1-t)$ so that

$$
x^{\prime}(t)(u(s(t))-x(t))+y^{\prime}(t)(v(s(t))-y(t))=2 t(1-t) \phi(t),
$$


where $\phi(t)$ is a cubic polynomial in Bézier form

$$
\begin{aligned}
\phi(t)= & \left.\left.\left(4 \Delta x_{0} F_{1}+\Delta x_{1} E_{2}^{2}\left(u_{0}-x_{0}\right)\right)+4 \Delta y_{0} G_{1}+\Delta y_{1} E_{2}^{2}\left(v_{0}-y_{0}\right)\right)\right) B_{0}^{3}(t) \\
& +\frac{1}{3}\left(6 \Delta x_{0} F_{2}+4 \Delta x_{1} F_{1}+6 \Delta y_{0} G_{2}+4 \Delta y_{1} G_{1}\right) B_{1}^{3}(t) \\
& +\frac{1}{3}\left(4 \Delta x_{0} F_{3}+6 \Delta x_{1} F_{2}+4 \Delta y_{0} G_{3}+6 \Delta y_{1} G_{2}\right) B_{2}^{3}(t) \\
& +\left(\Delta x_{0} E_{1}^{2}\left(u_{2}-x_{2}\right)+4 \Delta x_{1} F_{3}+\Delta y_{0} E_{1}^{2}\left(v_{2}-y_{2}\right)+4 \Delta y_{1} G_{3}\right) B_{3}^{3}(t) .
\end{aligned}
$$

Proposition 2.2. For $d<\min (1 / k(t))$, the Hausdorff distance between two curves $\mathbf{b}^{d}(t)$ and $\mathbf{p}(s)$ is equal to

$$
d_{H}\left(\mathbf{b}^{d}, \mathbf{p}\right)=\max _{0<t_{i}<1}\left|\mathbf{b}^{d}\left(t_{i}\right)-\mathbf{p}\left(s_{i}\right)\right|,
$$

where $t_{i} \in(0,1), i=1,2,3$, is the solution of the cubic equation $\phi(t)=0$ and $s_{i}=\frac{E_{1} t_{i}}{E_{2}\left(1-t_{i}\right)+E_{1} t_{i}} \in(0,1)$.

Proof. Since for all $t \in[0,1]$

$$
\mathbf{b}^{\prime}(t) \|\left(\mathbf{b}^{d}\right)^{\prime}(t), \quad \mathbf{b}^{\prime}(t) \perp\left(\mathbf{b}^{d}(t)-\mathbf{b}(t)\right),
$$

$(t, s)$ satisfy Equations (2.1) - (2.2) if and only if $(\mathrm{t}, \mathrm{s})$ satisfy

$$
\mathbf{p}^{\prime}(s) \|\left(\mathbf{b}^{d}\right)^{\prime}(t) \quad \text { and } \quad \mathbf{p}^{\prime}(s) \perp\left(\mathbf{p}(s)-\mathbf{b}^{d}(t)\right) .
$$

Also, the points $\mathbf{b}(t), \mathbf{b}^{d}(t)$ and $\mathbf{p}(s)$ are collinear. Thus the Hausdorff distance $d_{H}\left(\mathbf{b}^{d}, \mathbf{p}\right)$ is obtained from the maximum distance between $\mathbf{p}(s)$ and $\mathbf{b}^{d}(t)$ satisfying Equations (2.1)-(2.2). Since the solutions of Equations (2.1)(2.2) satisfy the cubic equation $\phi(t)=0$ and $s=E_{1} t /\left(E_{2}(1-t)+E_{1} t\right)$, the assertion follows.

\section{Example}

In this section we present an example for the $G C^{1}$ quadratic Bézier interpolation of the offset curve of the quadratic Bézier curve $\mathbf{b}(t)$ (thick lines in Figure 3) given by the control points

$$
\mathbf{b}_{0}=(0,0), \quad \mathbf{b}_{1}=(2,1), \quad \mathbf{b}_{2}=(2,0)
$$

with offset distance $d=-1$. By Proposition 2.1, the quadratic Bézier approximation $\mathbf{p}(s)$ (dotted lines) has the control points

$$
\begin{aligned}
& \mathbf{p}_{0}=(-1 / \sqrt{5}, 2 / \sqrt{5}), \quad \mathbf{p}_{1}=\left(2+\frac{-2 \sqrt{5}+5 \sqrt{2}}{10+\sqrt{10}}, 1+\frac{4 \sqrt{5}+5 \sqrt{2}}{10+\sqrt{10}}\right), \\
& \mathbf{p}_{2}=(2+1 / \sqrt{2}, 1 / \sqrt{2})
\end{aligned}
$$

as shown in Figure 3 .

$$
E_{1}=-3\left(1+\frac{3 \sqrt{2}}{10+\sqrt{10}}\right)=-3.967, \quad E_{2}=-3\left(1+\frac{3 \sqrt{5}}{10+\sqrt{10}}\right)=-4.529
$$




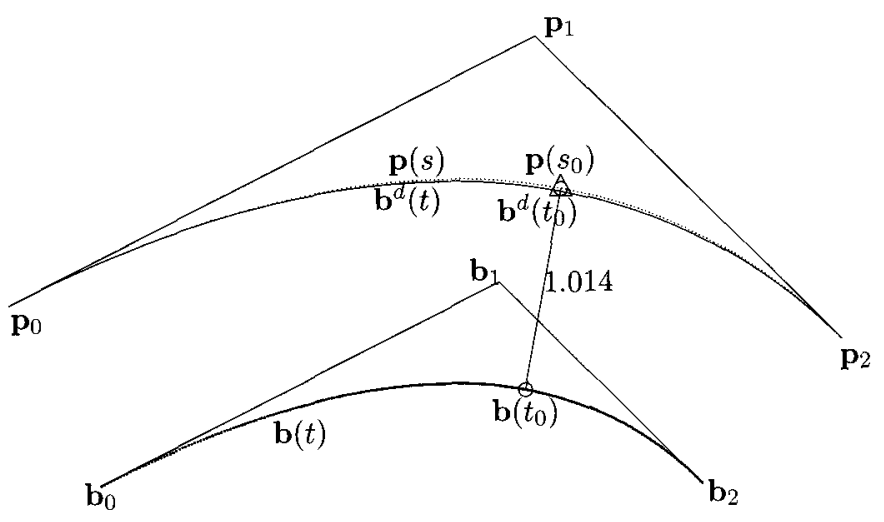

FIGURE 3. The distance between two points $\mathbf{p}\left(s_{0}\right)$ and $\mathbf{b}^{d}\left(t_{0}\right)$ (triangle) is 0.014 , which means that $d_{H}\left(\mathbf{p}, \mathbf{b}^{d}\right)=0.014$

$$
\begin{aligned}
s(t) & =\frac{(3 \sqrt{2}+10+\sqrt{10}) B_{1}^{1}(t)}{(3 \sqrt{5}+10+\sqrt{10}) B_{0}^{1}(t)+(3 \sqrt{2}+10+\sqrt{10}) B_{1}^{1}(t)} \\
& =\frac{-3.967 B_{1}^{1}(t)}{-4.529 B_{0}^{1}(t)+-3.967 B_{1}^{1}(t)}
\end{aligned}
$$

as shown in Figure 4. By Equations (2.3)-(2.4), we have

$$
\begin{array}{ccc}
F_{1}=-5.358, & F_{2}=.3036, & F_{3}=6.223 \\
G_{1}=18.83, & G_{2}=19.49, & G_{3}=17.61
\end{array}
$$

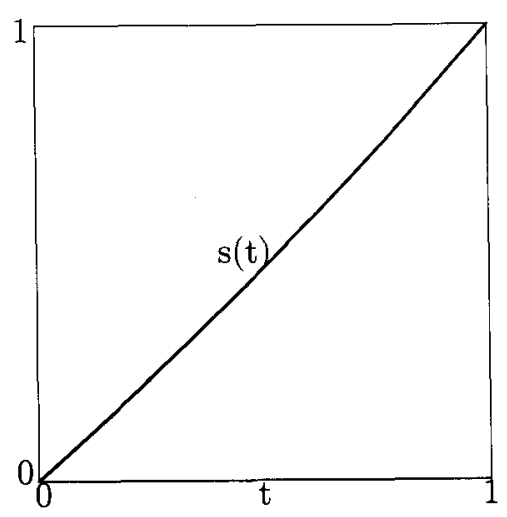

Figure 4. $s(t)$ (thick lines). 
and the cubic polynomial in Bézier form given by

$$
\phi(t)=9.883 B_{0}^{3}(t)+17.96 B_{1}^{3}(t)-55.32 B_{2}^{3}(t)+3.158 B_{3}^{3}(t)
$$

which has zeros at $t=-.2889, .6306$ and 17.17. The zero of $\phi(t)$ at $t_{0}=.6306$ is the unique solution of $\phi(t)=0$ inside the open interval $(0,1)$ combined with $s_{0}=s(.6306)=.5993$. Since distance between two points $\mathbf{b}(.6306)$ (small circle) and $\mathbf{p}(.5993)$ (small circle) is 1.014 as shown in Figure 3, finally we obtain the Hausdorff distance between two curve $\mathbf{b}^{d}(t)$ and its approximation curve $\mathbf{p}(s)$

$$
d_{H}\left(\mathbf{b}^{d}, \mathbf{p}\right)=0.014
$$

\section{References}

[1] Y. J. Ahn, Conic Approximation of planar curves, Comp. Aided Desi. 33 (2001), no. $12,867-872$.

[2] _ـ Helix approximation with conic and qadratic Bézier curves, Comp. Aided Geom. Desi. 22 (2005), no. 6, 551-565.

[3] Y. J. Ahn and H. O. Kim, Approximation of circular arcs by Bézier curves, J. Comp. Appl. Math. 81 (1997), 145-163.

[4] $\longrightarrow$, Curvatures of the quadratic rational Bézier curves, Comp. Math. Appl. 36 (1998), no. 9, 71-83.

[5] Y. J. Ahn, H. O. Kim, and K. Y. Lee, $G^{\mathbf{1}}$ arc spline approximation of quadratic Bézier curves, Comp. Aided Desi. 30 (1998), no. 8, 615-620.

[6] Y. J. Ahn, Y. S. Kim, and Y.S. Shin, Approximation of circular arcs and offset curves by Bezier curves of high degree, J. Comp. Appl. Math. 167 (2004), 181-191.

[7] G. Elber, I. K. Lee, and M. S. Kim, Comparing offset curve approximation method, IEEE Comp. Grap. Appl. 17 (1997), no. 3, 62-71.

[8] G. Farin, Curvature continuity and offsets for piecewise conics, ACM Tran. Grap. (1989), 89-99.

[9] _ Curves and Surfaces for Computer Aided Geometric Design, Academic Press, San Diego, CA, 1993.

[10] R. T. Farouki, Conic Approximation of Conic Offsets, J. Symb. Comp. 23 (1997), 301313.

[11] R. T. Farouki and J. K. Johnstone, The bisector of a point and a plane parametric curve, Comp. Aided Geom. Desi. 11 (1994), no. 2, 117-151.

[12] R. T. Farouki and C. A. Neff, Analytic properties of plane offset curves, Comp. Aided Geom. Desi. 7 (1990), 83-99.

[13] R. T. Farouki and T. W. Sederberg, Analysis of the offset to a parabola, Comp. Aided Geom. Desi. 8 (1995), 639-645.

[14] S. H. Kim and Y. J. Ahn, Approximation of circular arcs by quartic Bezier curves, Comp. Aided Desi. 39 (2007), no. 6, 490-493.

[15] I. K. Lee, M. S. Kim, and G. Elber, Planar curve offset based on circle approximation, Comp. Aided Desi. 28 (1996), 617-630.

Department of Mathematics EduCATion

CHOSUN UNIVERSITY

GWANGJU 501-759, KorEA

E-mail address: ahn@chosun.ac.kr 